
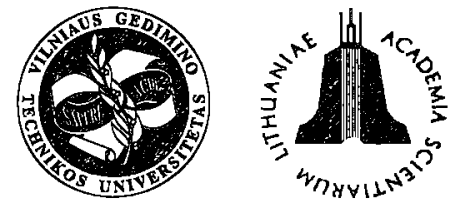

ISSN 1648-4142 TRANSPORT

http:/www.vtu.1t/english/editions

TRANSPORT - 2003, Vol XVIII, No 3, 136-142

\title{
A MATHEMATICAL MODEL OF THE VIBRATING SYSTEM EQUIVALENT TO THE VEHICLE IN THE MODE OF EMERGENCY BRAKING
}

\author{
Robertas Pečeliūnas $^{1}$, Ona Lukoševičiené ${ }^{2}$, Olegas Prentkovskis ${ }^{3}$ \\ 1,2 Dept of Automobile Transport, Vilnius Gediminas Technical University, \\ J. Basanavičiaus g. 28, LT-2009 Vilnius, Lithuania \\ ${ }^{3}$ Dept of Transport Technological Equipment, Vilnius Gediminas Technical University, \\ Plytines g. 27, LT-2016 Vilnius, Lithuania
}

Received 200301 10; accepted 20030430

\begin{abstract}
A mathematical model of the vibrating system equivalent to the vehicle in the mode of emergency braking is developed and the solution is offered. The problem associated with the comparison of the vibrations of the braking vehicle with the oscillations of a uniformly translating vehicle is considered. On the basis of the analysis of the available research data some assumptions and constraints are introduced, allowing the vibrating system equivalent to the condition of the vehicle in the mode of emergency braking to be developed. The system obtained differs from the data found in the theory of the vehicle in the description of the applied tangential forces caused by the force of inertia applied to the center of gravity. Based on the equivalent system developed, the differential equations describing the displacements of masses of the above system are derived and solved.
\end{abstract}

Keywords: mathematical model; dynamics of the vehicle; equivalent vibrating system; flexible mounted and inflexible mounted vehicle masses; normal deformation; static equilibrium; deceleration; the moment of the tangential force.

\section{Introduction}

A motor vehicle may be looked upon as a system consisting of particular masses (i. e. the body, axles, wheels, etc.). These separate masses (parts) may displace with respect to each other, rotate or change their position in some other way. This is especially relevant for studying the vehicle dynamics (for example, in breaking).

When a vehicle is subjected to the action of transverse and longitudinal forces, the axles and right and left side wheels are more heavily loaded on one side of the vehicle then on the other, therefore its body may be displaced or turned.

The following types of displacements are practically important [1]:

- linear vertical vibrations of the body (swinging);

- angular vibrations of the body in the longitudinal

plane of the automobile (jumping);

- angular vibrations of the body in the transverse plane of the automobile (rocking).

The EC Council Directive 71/320 [2] emphasizes that the vehicle should be designed to be able to keep the rectilinear translation in case of emergency braking.

\footnotetext{
1 E-mail: robec@ti.vtu.lt

${ }^{2}$ E-mail: vaida@ti.vtu.lt

${ }^{3}$ E-mail: olegas@ti.vtu.lt
}

However, the authors of the present investigation are concerned with the problem of redistribution of the particular masses in a vehicle which is slowing down during the emergency braking.

\section{Major Assumptions and Constraints}

The study of literature on the problem [3-10] has shown that to develop a mathematical model of the process considered, some assumptions have to be made, allowing for the comparison of vehicle vibrations. The task is to compare the vibrations occurring in emergency braking with those found when a vehicle is in the mode of uniform translation. In order to identify the differences of the above types of vibration, maintaining the equivalent vibrating system at the same level of complexity as found in the theory of uniform translation and vehicle suspension, the particular relationships should not be taken into account or evaluated. The latter refer to some minor kinematic relations of the revolving wheels and the supporting frame (plane). Moreover, the approach suggested allows the assumptions introduced in developing the vibration model of a braking vehicle to be more clearly grounded.

This approach to developing a mathematical vibration model of the vehicle taking into account the treatment of similar problems by other authors determine the 
following constraints and assumptions adopted in the present investigation:

1. Taking into account that phase 1 and phase 2 of braking considered in [10] make only a fraction of the whole process, it is assumed that at these stages the process of braking follows the same path as determined for the main phase 3 . In other words, it is assumed that the wheels of the vehicle are brought to a stop as soon as the brakes are applied.

2. The coupling coefficient between the tyre and the surface of the road pavement is independent of the speed, with its instability being assessed statically by an average value of the coupling coefficient of braking on the road.

3. Since only vertical vibrations in the longitudinal plane are considered for the vehicle with non rotating wheels when the brakes are applied, the tangential stiffness of the kinematic contacts of tyre and pavement is assessed together with the normal stiffness.

4. The equivalent vibrating system of the vehicle is considered in a longitudinal plane assuming that the road pavement symmetrically acts on the right and left side wheels of the vehicle. Moreover, all forces and moments occurring in the suspension due to tangential braking forces in the zone of tyre and road contact are assumed to be the responses of a guiding lever. The guide provides only for one vertical displacement freedom of the axle and the flexible mounted mass.

5. The equivalent vibrating system of the vehicle is reduced to a system with concentrated masses and linear characteristics of recovering and damping forces and the deformation functions and their derivatives of all the elastic elements and dampers.

6 . In the equivalent vibrating system some external factors such as air resistance, road microprofile gradient or downgrade, etc. are not taken into account as it is commonly done in the theory of vehicle when the dynamics of braking is handled.

Hence, it leads us to conclude that the equivalent vehicle vibrating system to be used in modelling the vibrations of a braking automobile differs from the system commonly used in the theory of vehicle vibration and uniform translation $[5,6]$ only by tangential forces acting under the interaction of the wheels and road pavement and causing the force of inertia to act on the center of gravity.

\section{The Equivalent Vibrating System of the Vehicle}

The vibrating system equivalent to the vehicle in the braking mode is schematically shown in Fig 1.

The diagram is drawn taking into account the expressions commonly used in the theory of uniform vehicle translation and the assumptions made, as well as using the following notation:

$M$ - flexible mounted mass;
$I_{Y}$ - inertia moment of a flexible mounted mass, depending on central cross axle $0 ; m_{1,2}$ - inflexible mounted masses of the front and rear axles; $c_{s p 1,2}-$ stiffness coefficients of the front and rear suspension springs; $\eta_{1,2}-$ proportionality coefficients of the deformation velocity and inelastic forces of dampers, springs and suspension; $c_{p 1,2}$ - normal stiffness coefficient of the tyre; $q_{1,2}$ - the values of the ordinates of the road surface microprofile with respect to time during the contact with the front and rear wheels; $\xi_{1,2}-$ vertical displacements of inflexible mounted masses of suspensions; $z_{1,2}$-vertical displacements of flexible mounted masses of suspensions; $z_{0}-$ vertical displacements of the center of gravity denoted by 0 of a flexible mounted mass; $\alpha$ angular displacements of a flexible mounted mass about the center of gravity; $P_{\tau 1,2}$ - the tangential forces of the road in contact with the front wheels of a braking vehicle; $F_{j}$ - the force of inertia of the total mass of a braking vehicle; $r_{1,2}-$ a couple of forces equivalent to the moment of tangential forces and inertia; $L$-base of a vehicle; $a, b$ - the distance from the center of gravity to the vertical planes of the front and rear inflexible mounted masses; $h_{c}$-the height of the vehicle center of gravity up to the middle line of road surface.

Indices 1,2 of the vibrating system equivalent to the vehicle in emergency braking denote that the parameters refer to the front or rear suspension or are valid in the vertical plane passing through the front and rear wheel axles of the vehicle. The coordinates of the vehicle masses in the vertical plane are determined in terms of the time function $t$ with respect to the static equilibrium. Moreover, the increase of the equilibrium height $h_{c}$ over the middle line of road pavement microprofile is considered negligible compared with its value of coordinates defining the location of a flexible mounted mass at the moment. Their interrelationship may be expressed in the following way:

$$
z_{0}=\frac{z_{2} a+z_{1} b}{a+b} ; \alpha=\frac{z_{1}-z_{2}}{a+b} .
$$

Then, when the variables are differentiated with respect to time, the values with respect to their argument are denoted by a dot over a variable symbol.

In order to derive the equilibrium equations of the masses of the system in motion the relationship between the forces of inertia and the equivalent vertical forces in the suspension plane should be considered first.

For example, if the swinging of the vehicle [10] is considered, the total moment acting on the flexible mounted mass due to braking forces under the conditions specified is equal to:

$$
M_{r}=\left(P_{\tau 1}+P_{\tau 2}\right) h_{c} .
$$

The effect of the above moment is practically the same as the action of the force couple $r_{1}=r_{2}$, having an arm equal to the base of the vehicle. Hence:

$$
M_{r}=\left(P_{\tau 1}+P_{\tau 2}\right) h_{c}=r L,
$$




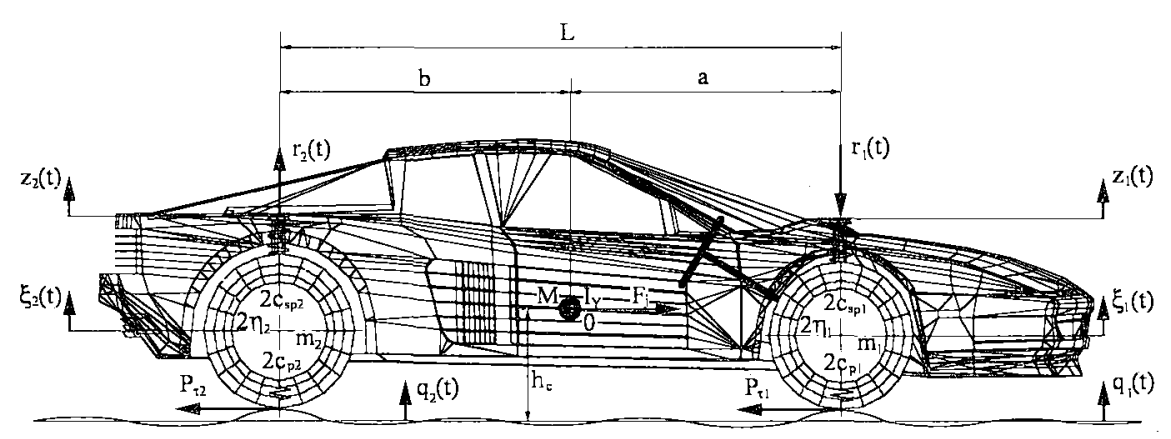

a)

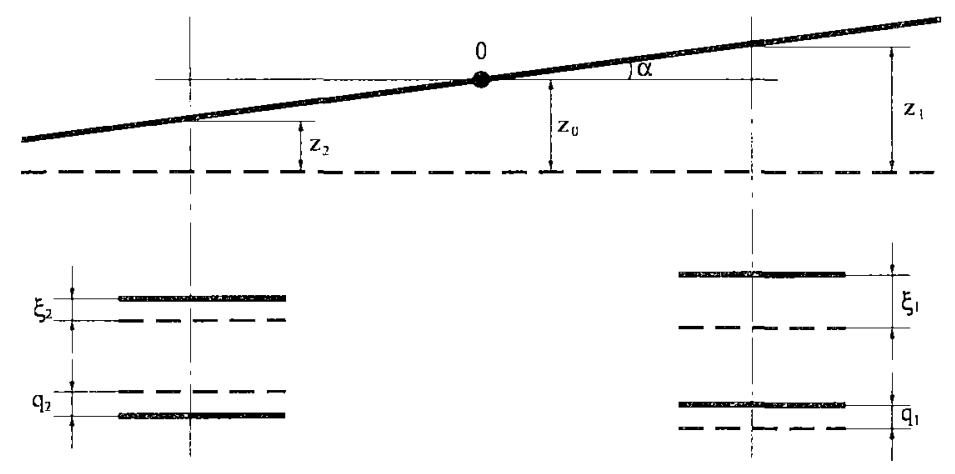

b)

Fig 1. A schematic view of the vibrating system equivalent to the vehicle in the braking mode: a - basic parameters and coordinates; $b$ - interrelations of coordinates at any moment of time (dotted line shows the state of equilibrium)

therefore,

$$
r=\left(P_{\tau 1}+P_{\tau 2}\right)_{L}^{h_{c}}
$$

Braking forces acting on the wheels cause the vertical forces proportional to their sum, acting on the vertical flexible mounted mass in the vertical planes of the front and rear suspensions in an opposite direction as shown in the diagram.

It should be emphasized that the expression (2) is valid only for the assumption that the guide of the suspension ensures the transfer of the longitudinal forces applied to the wheels to the vehicle body on account of the internal forces, while the suspension kinematics is independent of the relative vertical displacements of the points of fixing the springs and flexible mounted or inflexible mounted masses.

According to Fig 1, with the vibrations in the plane of the drawing, the flexible mounted mass is acted upon by the following forces, in addition to the force couple $r$ moment equivalent to the inertia moment of a braking vehicle: the force of inertia for the vertical displacements of the center of gravity $P_{m}$, vertical resisting forces $P_{\eta 1,2}$ of inelastic springs and dampers, elastic spring forces $P_{s p 1,2}$ and their moments with respect to the center of gravity as well as the inertia moment $M_{i}$ of the flexible mounted mass when the latter revolves about the cross axle passing through the center of gravity. These forces and moments are expressed in terms of the coordinates of the masses at the moment of time, their derivatives and the proportionality coefficients of the elastic and inelastic suspensions.

\section{Differential Equations of Mass Motion of the Equivalent Vibrating System}

When deriving the equations for the equilibrium of masses with all the above described forces acting the following symbols were introduced:

$$
\Omega_{1,2}=\sqrt{\frac{2 c_{s p 1,2}}{M_{1,2}}}, \omega_{1,2}=\sqrt{\frac{2 c_{p 1,2}}{m_{1,2}}} \text {-natural vibra- }
$$

tion frequencies of the front and the rear flexible mounted and inflexible mounted masses found on the springs and tyres;

$$
k_{1,2}=\frac{\eta_{1,2}}{M_{1,2}}-\text { coefficients of inelastic resistance }
$$

of the front and rear suspensions;

$$
\begin{aligned}
& \mu_{1,2}=\frac{m_{1,2}}{M_{1,2}} ; r_{1,2}=\stackrel{r}{M_{1,2}} ; \\
& \chi_{1,2}=\frac{M_{3}}{M_{1,2}} ; M_{3}=M \frac{a b-\rho_{y}^{2}}{L^{2}} .
\end{aligned}
$$


Having evaluated the above given expressions and combined the equilibrium equations, it is possible to write the systems of the second order differential equations describing the vibrations of the braking vehicle masses.

For strong inertial relation between the vibrations of the flexible mounted front and rear masses the following equations are obtained:

$$
\left\{\begin{array}{l}
M_{1} \ddot{z}_{1}+M_{3} \ddot{z}_{2}+2 \eta_{1}\left(\dot{z}_{1}-\dot{\xi}_{1}\right)+2 c_{s p 1}\left(z_{1}-\xi_{1}\right)+r_{1}=0 \\
M_{2} \ddot{z}_{2}+M_{3} \ddot{z}_{1}+2 \eta_{2}\left(\dot{z}_{2}-\dot{\xi}_{2}\right)+2 c_{s p 2}\left(z_{2}-\xi_{2}\right)+r_{2}=0 \\
m_{1} \ddot{\xi}_{1}+2 c_{p 1}\left(\xi_{1}-q_{1}\right)-2 \eta_{1}\left(\dot{z}_{1}-\dot{\xi}_{1}\right)-2 c_{s p 1}\left(z_{1}-\xi_{1}\right)=0 \\
m_{2} \ddot{\xi}_{2}+2 c_{p 2}\left(\xi_{2}-q_{2}\right)-2 \eta_{2}\left(\dot{z}_{2}-\dot{\xi}_{2}\right)-2 c_{s p 2}\left(z_{2}-\xi_{2}\right)=0,
\end{array}\right.
$$

or introducing the conditional notation values we get:

$$
\left\{\begin{array}{c}
\ddot{z}_{1}+\chi_{1} \ddot{z}_{2}+2 k_{1}\left(\dot{z}_{1}-\dot{\xi}_{1}\right)+\Omega_{1}^{2}\left(z_{1}-\xi_{1}\right)=\left(-1 / M_{1}\right) r_{1} \\
\ddot{z}_{2}+\chi_{2} \ddot{z}_{1}+2 k_{2}\left(\dot{z}_{2}-\dot{\xi}_{2}\right)+\Omega_{1}^{2}\left(z_{2}-\xi_{2}\right)=\left(1 / M_{2}\right) r_{2} \\
\ddot{\xi}_{1}+\omega_{1}^{2} \xi_{1}-2 \frac{k_{1}}{\mu_{1}}\left(\dot{z}_{1}-\dot{\xi}_{1}\right)-\frac{\Omega_{1}^{2}}{\mu_{1}}\left(z_{1}-\xi_{1}\right)=\omega_{1}^{2} q_{1} \\
\ddot{\xi}_{2}+\omega_{2}^{2} \xi_{2}-2 \frac{k_{2}}{\mu_{2}}\left(\dot{z}_{2}-\dot{\xi}_{2}\right)-\frac{\Omega_{2}^{2}}{\mu_{2}}\left(z_{2}-\xi_{2}\right)=\omega_{2}^{2} q_{2} .
\end{array}\right.
$$

If the inertial relation between the front and rear mass vibrations is not observed, the equation (4) is of the form:

$$
\left\{\begin{array}{l}
\ddot{z}_{1}+2 k_{1}\left(\dot{z}_{1}-\dot{\xi}_{1}\right)+\Omega_{1}^{2}\left(z_{1}-\xi_{1}\right)=-v_{1} r_{1} \\
\ddot{\xi}_{1}+\omega_{1}^{2} \xi_{1}-2 \frac{k_{1}}{\mu_{1}}\left(\dot{z}_{1}-\dot{\xi}_{1}\right)-\frac{\Omega_{1}^{2}}{\mu_{1}}\left(z_{1}-\xi_{1}\right)=\omega_{1}^{2} q_{1} \\
\ddot{z}_{2}+2 k_{2}\left(\dot{z}_{2}-\dot{\xi}_{2}\right)+\Omega_{2}^{2}\left(z_{2}-\xi_{2}\right)=-v_{2} r_{2} \\
\ddot{\xi}_{2}+\omega_{2}^{2} \xi_{2}-2 \frac{k_{2}}{\mu_{2}}\left(\dot{z}_{2}-\dot{\xi}_{2}\right)-\frac{\Omega_{2}^{2}}{\mu_{2}}\left(z_{2}-\xi_{2}\right)=\omega_{2}^{2} q_{2} .
\end{array}\right.
$$

In dealing with the theory of vehicle suspension and uniform translation the problem of the analysis of the elastic elements (springs and tyres), the deformation rather than the variations of the coordinates and their derivatives (i. e. the acceleration of the flexible mounted mass at the points $\ddot{z}_{1}$ and $\ddot{z}_{2}$ of the vertical vibrations $z_{1}$ and $z_{2}$ may often arise. Because of this fact, having made some transformations, we consider relative rather than absolute mass displacement of the system:

$x_{1,2}=z_{1,2}-\xi_{1,2}-$ the instantaneous value of the deflection of the springs;

$y_{1,2}=\xi_{1,2}-q_{1,2}$ - the instantaneous value of normal tyre deformation.

Hence:

$$
\begin{aligned}
& z_{1,2}=x_{1,2}+y_{1,2}+q_{1,2} ; \\
& \ddot{z}_{1,2}=\ddot{x}_{1,2}+\ddot{y}_{1,2}+\ddot{q}_{1,2} ;
\end{aligned}
$$

$$
\dot{x}_{1,2}=\dot{z}_{1,2}-\dot{\xi}_{1,2} \text {. }
$$

Substituting the above expressions into (4) the system of equations for strong inertial relation between the front and rear parts of the flexible mounted mass, may be written as follows:

$$
\left\{\begin{array}{l}
\ddot{x}_{1}+\ddot{y}_{1}+\chi_{1} \ddot{x}_{2}+\chi_{1} \ddot{y}_{2}+2 k_{1} \dot{x}_{1}+\Omega_{1}^{2} x_{1}=-v_{1} r-\ddot{q}_{1}-\chi_{1} \ddot{q}_{2} \\
\ddot{x}_{2}+\ddot{y}_{2}+\chi_{2} \ddot{x}_{1}+\chi_{2} \ddot{y}_{1}+2 k_{2} \dot{x}_{2}+\Omega_{2}^{2} x_{2}=-v_{2} r-\ddot{q}_{2}-\chi_{2} \ddot{q}_{1} \\
\ddot{y}_{1}+\omega_{1}^{2} y_{1}-2 \frac{k_{1}}{\mu_{1}} \dot{x}_{1}-\frac{\Omega_{1}^{2}}{\mu_{1}} x_{1}=-\ddot{q}_{1} \\
\ddot{j}_{2}+\omega_{2}^{2} y_{2}-2 \frac{k_{2}}{\mu_{2}} \dot{x}_{2}-\frac{\Omega_{2}^{2}}{\mu_{2}} x_{2}=-\ddot{q}_{2} .
\end{array}\right.
$$

Here, similar to the equality (5), the following notation is introduced:

$$
v_{1}=\frac{1}{M_{1}} \text { and } v_{2}=\frac{1}{M_{2}} \text {. }
$$

When the inertial relations are insignificant, we may write the following expression substituting (5) into (6):

$$
\left\{\begin{array}{l}
\ddot{x}_{1}+\ddot{y}_{1}+2 k_{1} \dot{x}_{1}+\Omega_{1}^{2} x_{1}=-v_{1} r_{1}-\ddot{q}_{1} \\
\ddot{y}_{1}+\omega_{1}^{2} y_{1}-2{ }_{\mu_{1}}^{k_{1}} \dot{x}_{1}-\Omega_{1}^{2} x_{1}=-\ddot{q}_{1} \\
\ddot{x}_{2}+\ddot{y}_{2}+2 k_{2} \dot{x}_{2}+\Omega_{2}^{2} x_{2}=-v_{2} r_{2}-\ddot{q}_{2} \\
\ddot{y}_{2}+\omega_{2}^{2} y_{2}-2 \frac{k_{2}}{\mu_{2}} \dot{x}_{2}-\frac{\Omega_{2}^{2}}{\mu_{2}} x_{2}=-\ddot{q}_{2} .
\end{array}\right.
$$

To any of the system equation the condition of braking forces formation, and, evidently, the force of inertia and the force couple $r$ equivalent to them, belonging to differential equations, are added. Besides, three major features of their formation should be analytically expressed.

The first one means that braking forces at any moment of time are proportional to normal forces (responses), while for the linear elasticity characteristic of tyres, the above forces are proportional to tyre deformations due to mass vibrations on a rough surface. This proportionality is assessed by the coupling coefficient of tyres and road pavement.

The normal values of deformations denoted by $y_{1}$ and $y_{2}$ in differential equations are counted off from the static equilibrium as found in the theory of uniform translation and shock proofing. To express the values of the instantaneous braking forces in terms of normal forces (responses), it is necessary to assess the total deforma- 
tion of tyres, taking the initial contact of tyres with the pavement as the point of departure. This follows from a physical meaning of braking, implying that when the brakes are applied, $y_{1}=y_{2}=0$, the force of inertia and the equivalent force couple $r$ immediately come into play. Based on the above statement, the equality (2) may be written in the following way for any moment after braking the wheels:

$$
\begin{aligned}
& r=\frac{h_{c}}{L}\left(P_{\tau 1}+P_{\tau 2}\right)=\frac{h_{c}}{L} \varphi\left(P_{N 1}+P_{N 2}\right)= \\
& \frac{h_{c}}{L} \varphi\left(2 c_{p 1} y_{n 1}+2 c_{p 2} y_{n 2}\right)= \\
& h_{c} \varphi\left(2 c_{p 1}\left(y_{s t 1}+y_{1}\right)+2 c_{p 2}\left(y_{s t 2}+y_{2}\right)\right)= \\
& \frac{h_{c}}{L} \varphi\left(\left(2 c_{p 1} y_{s t 1}+2 c_{p 2} y_{s t 2}\right)+\left(2 c_{p 1} y_{1}+2 c_{p 2} y_{2}\right)\right)= \\
& \frac{h_{c}}{L} \varphi\left(M g+\left(2 c_{p 1} y_{1}+2 c_{p 2} y_{2}\right)\right) .
\end{aligned}
$$

here: $P_{N 1,2}$ - instantaneous values of normal forces during the contact of the wheels and pavement, $y_{n 1,2}-$ maximum normal tyre deformations, $y_{s t 1,2}$ - tyre deformations for the vehicle in static equilibrium on a horizontal road.

Thus the force $r$, being a violating factor in the differentiation equations, is expressed in terms of the instantaneous value deviation of the normal deformations of tyres from the constant level corresponding to the static load.

The second peculiarity found in the analytical description of the formation of the forces $r$ refers to the instantaneous addition of the statistical component to the obtained expression (9). In order to give the analytical description of the above phenomenon it is necessary to match the beginning of time counting with the time of initial braking, using the Heaviside unit function $\delta_{0}(t)$, expressing a single jump:

$$
\delta_{0}(t)=\left\{\begin{array}{l}
0, \text { when } t<0 \\
1, \text { when } t>0 .
\end{array}\right.
$$

Then, multiplying the above function by the static component of the braking force and determining that in braking $y_{1}$ and $y_{2}$ become the time functions due to the occurring mass vibration of the system, the forces $r$ may also be expressed for any moment of time in this form:

$$
r(t)=\frac{h_{c}}{L} \varphi\left(M q \delta_{0}(t)+2 c_{p 1} y_{1}+2 c_{p 2} y_{2}\right) .
$$

For brevity, the following notation is introduced:

$$
{ }_{L}^{h_{c}} \varphi M q=a_{0} ; 2 c_{p 1} \varphi \frac{h_{c}}{L}=a_{1} ; 2 c_{p 2} \varphi \frac{h_{c}}{L}=a_{2} .
$$

Thus, we finally get:

$$
r(t)=a_{0} \delta_{0}(t)+a_{1} y_{1}+a_{2} y_{2} .
$$

The third peculiarity of the forces $r(t)$ formation is associated with the fact that the braking of the vehicle may take place at any time, when the vehicle is uniformly translating along the rough road. The flexible mounted and inflexible mounted masses, being in the state of continuous vibration at the time of the emergence of the braking forces $(t=0)$, acquire a certain defined state and speed determined by the coordinates. To emphasize these instantaneous values at the time of occurrence of braking forces and responses, the zero symbols may be introduced into the notation index.

Then, it should be assumed that:

$$
\begin{aligned}
& z_{1,2}=z_{01,2} ; \dot{\xi}_{1,2}=\xi_{01,2} ; q_{1,2}=q_{01,2} ; \\
& \dot{z}_{1,2}=\dot{z}_{01,2} ; \dot{\xi}_{1,2}=\dot{\xi}_{01,2} ; \dot{q}_{1,2}=\dot{q}_{01,2}, \text { when } t=0 .
\end{aligned}
$$

Taking into account the above statements, the following initial conditions should be evaluated in further consideration of the vehicle vibrations, when $t>t_{0}$. When the effect of the rough road surface on the moving vehicle is described in terms of a random stationary process with the normal distribution law, the initial conditions are described statistically in terms of random values also having normal distribution. This follows from the condition that the equivalent system is not varying statistically. When the brakes are applied, the respective values of the distribution of the initial conditions of the vehicle oscillations (i. e. the average value, dispersion of the coordinates and their derivatives) are found on the basis of the microprofile effect spectral density and the system output parameters corresponding to the transfer functions, which are in the state when the coordinates of the mass position or their combinations are changing. In addition, the transfer functions are found from the same differential equations (7) and (8), but only when $r=0$. The exact calculations of the transfer functions of the systems equivalent to the vertically vibrating vehicle in a state of uniform translation are presented in the papers $[5,7]$. It should be noted that when the initial conditions are assessed, the forces $r$ at the initial moment will depend on the position of masses in braking.

The initial conditions are expressed as follows:

$$
z_{01,2}=\dot{z}_{01,2}=\xi_{01,2}=\dot{\xi}_{01,2}=q_{01,2}=\dot{q}_{01,2}=0,
$$

or

$$
x_{01,2}=\dot{x}_{01,2}=y_{01,2}=\dot{y}_{01,2}=0 \text {, when } t=0 \text {. }
$$


The physical meaning of the above conditions is that braking and blocking of the wheels take place at the moment when the system occasionally reaches the state of constant static equilibrium. According to the theory of probability, this is hardly possible though it may occur if zero values of a set of eight statistically distributed values coincide in time. This case is well - suited for theoretical research, when the systems equivalent to the translation of the vehicle with the wheels stopped on the rough road helps to more clearly define the transition process determined by suddenly emerging braking forces.

The valuable theoretical description of emergency braking considered is finally reduced to the description of the tangential force change in time:

$$
P_{\tau}=\varphi\left(\delta_{0}(t) P_{z}+\Delta P_{z}(t)\right) ;
$$

here: $P_{z}$ - the normal force of the road on the wheels equal to the static load; $\Delta P_{z}(t)-$ dynamic increase of the normal force due to vibration of flexible mounted and inflexible mounted masses.

\section{Computer Aided Test Results}

The system of differential equations (5) is considered. To solve it, the Euler method [11] is chosen:

$$
x_{t}=x_{t-1}+\Delta t f_{t-1}
$$

here: $x_{t}$ - the unknown at the time $t ; x_{t-1}$ - the unknown at the time $t-1 ; \Delta t$-integration step; $f_{t-1}$ - the right hand side of the $1^{\text {st }}$ order differential equation.

The differential equations are of the second order in the equation system (5). Before applying the Euler's method it should be transformed from 4 second-order differential equations into 8 first-order equations in this way:

$$
\left\{\begin{array}{l}
\frac{d z_{1}}{d t}=\dot{z}_{1} \\
\frac{d \dot{z}_{1}}{d t}=-v_{1} r_{1}-2 k_{1}\left(\dot{z}_{1}-\dot{\xi}_{1}\right)-\Omega_{1}^{2}\left(z_{1}-\xi_{1}\right) \\
\frac{d \xi_{1}}{d t}=\dot{\xi}_{1} \\
\frac{d \dot{\xi}_{1}}{d t}=\omega_{1}^{2} q_{1}-\omega_{1}^{2} \xi_{1}+2 \frac{k_{1}}{\mu_{1}}\left(\dot{z}_{1}-\dot{\xi}_{1}\right)+\frac{\Omega_{1}^{2}}{\mu_{1}}\left(z_{1}-\xi_{1}\right) \\
\frac{d z_{2}}{d t}=\dot{z}_{2} \\
\frac{d \dot{z}_{2}}{d t}=-v_{2} r_{2}-2 k_{2}\left(\dot{z}_{2}-\dot{\xi}_{2}\right)-\Omega_{2}^{2}\left(z_{2}-\xi_{2}\right) \\
\frac{d \xi_{2}}{d t}=\dot{\xi}_{2} \\
\frac{d \dot{\xi}_{2}}{d t}=\omega_{2}^{2} q_{2}-\omega_{2}^{2} \xi_{2}+2 \frac{k_{2}}{\mu_{2}}\left(\dot{z}_{2}-\dot{\xi}_{2}\right)+\frac{\Omega_{2}^{2}}{\mu_{2}}\left(z_{2}-\xi_{2}\right) .
\end{array}\right.
$$

The following unknowns are found in the system of differential equations: $z_{1}, \dot{z}_{1}, \xi_{1}, \dot{\xi}_{1}, z_{2}, \dot{z}_{2}, \xi_{2}, \dot{\xi}_{2}$.

A standard car possessing the following characteristics was tested:

$M=950 \mathrm{~kg} ; \quad M_{1}=600 \mathrm{~kg} ; \quad M_{2}=350 \mathrm{~kg} ;$ $m_{1}=70 \mathrm{~kg} ; \quad m_{2}=80 \mathrm{~kg} ; \quad a=1,38 \mathrm{~m} ; \quad b=1,29 \mathrm{~m} ;$ $h_{c}=0,52 \mathrm{~m} ; \quad c_{s p 1,2}=34 \mathrm{kN} / \mathrm{m} ; c_{p_{1,2}}=120 \mathrm{kN} / \mathrm{m}$; $\eta_{1}=5,34 \cdot 10^{3} \mathrm{~kg} / \mathrm{s} ; \eta_{2}=7,06 \cdot 10^{3} \mathrm{~kg} / \mathrm{s}$.

The braking is performed on an even road, i. e. $q_{1}=0, q_{2}=0$.

The application programs (software) were developed in Compaq Visual Fortran Professional v 6.1 [12] for the computer aided test.

The dependences of the displacements of masses $z_{1}, z_{2}, \xi_{1}, \xi_{2}$ and the velocities of masses $\dot{z}_{1}, \dot{z}_{2}, \dot{\xi}_{1}$, $\dot{\xi}_{2}$ on time are given in Fig $2-5$.

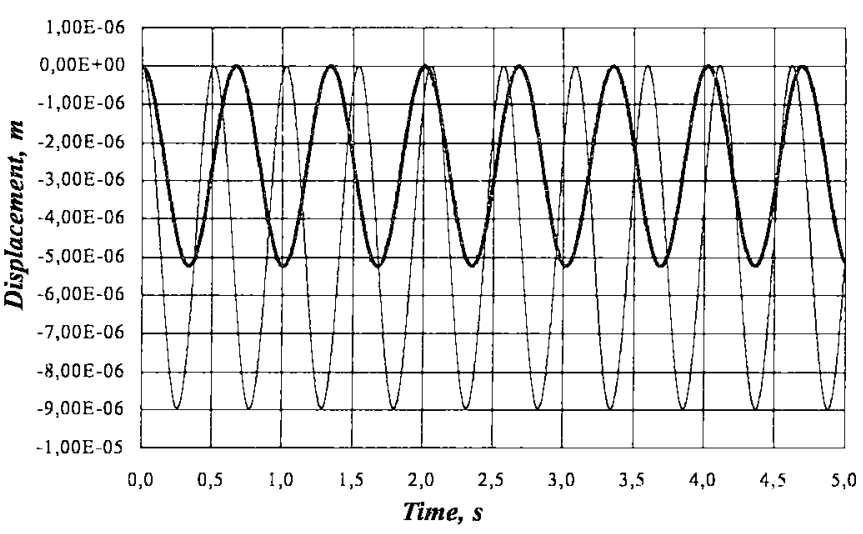

Fig 2. Vertical displacements of flexible mounted masses of the front and rear suspensions:

$z_{1}$ - heavy line; $z_{2}-$ thin line

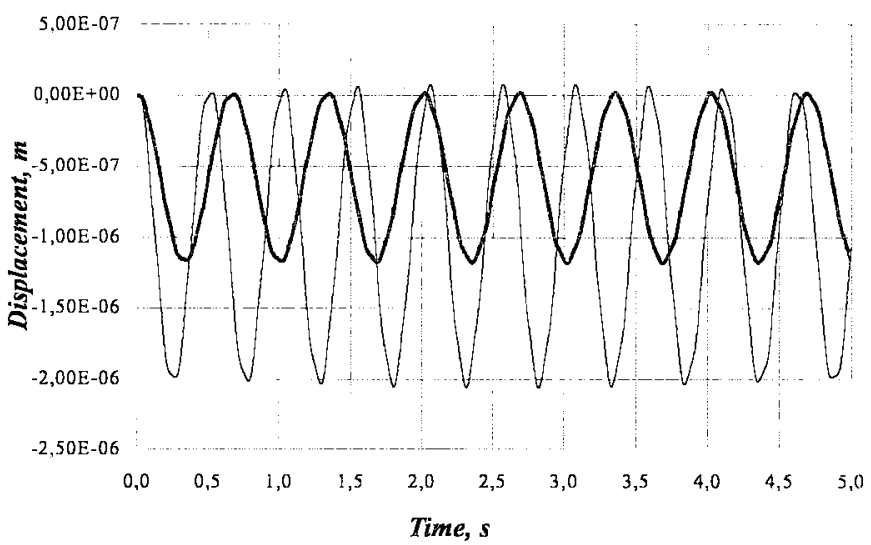

Fig 3. Vertical displacements of inflexible mounted masses of the front and rear suspensions: $\xi_{1}$ - heavy line; $\xi_{2}-$ thin line 


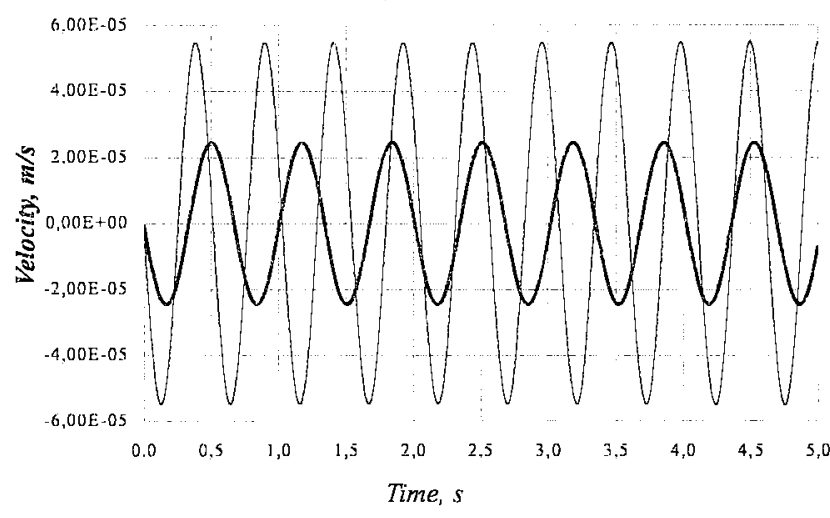

Fig 4. Vertical velocities of flexible mounted masses of the front and rear suspensions:

$\dot{z}_{1}$ - heavy line; $\dot{z}_{2}-$ thin line

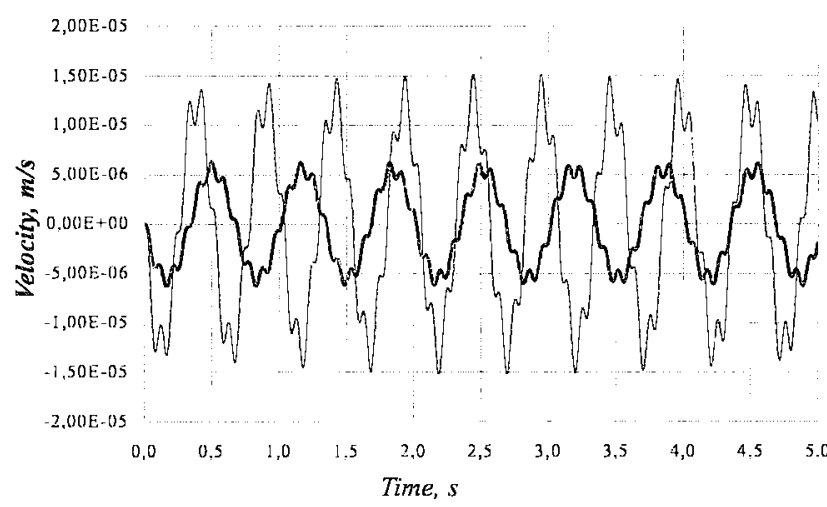

Fig 5. Vertical velocities of inflexible mounted masses of the front and rear suspensions: $\dot{\xi}_{1}$ - heavy line; $\dot{\xi}_{2}-$ thin line

\section{Conclusions}

1. A mathematical model of a vibrating system equivalent to the case of emergency braking vehicle was developed, allowing the position of the car body in an emergency to be more accurately defined.

When the brakes are applied, the vehicle masses are redistributed between the front and rear axles (the front of the vehicle bends over the road surface, while the rear part is lifted). This case is illustrated by the relationships given in Figs $2-5\left(z_{2}>z_{1}\right.$ and $\left.\xi_{2}>\xi_{1}\right)$.

3. The systems of differential equations developed allow us to analyze the unstable transition modes of braking the car on the even and rough road. By means of these equations it is possible to assess the impact of the initial state of the vehicle at the time of brake application (mass displacements and velocities) on the above processes.

4. The technique developed may be practically applied to technical expertise as well as in testing the dynamics of the vehicle and examining the mechanism of collision in the mode of emergency braking depending on the acceleration.

5. The software for solving the obtained mathematical model of the equivalent vibrating system for the vehicle in case of emergency braking was developed in Compaq Visual Fortran Professional v 6.1.

\section{References}

1. Margolis, D.; Shim, T. A bond graph model incorporating sensors, actuators, and vehicle dynamics for developing controllers for vehicle safety. Journal of the Franklin Institute, Vol 338, 2001, p. 21-34.

2. Council Directive $71 / 320 / \mathrm{EEC}$ on the approximation of the laws of the Member States relating to the braking devices of certain categories of motor vehicles and their trailers. Brussel, 1998.

3. Borner, M.; Straky, H.; Weispfenning, T.; Isermann, R. Model based fault detection of vehicle suspension and hydraulic brake system. Mechatronics, Vol 12, 2002, p. 999 1010.

4. Masao, Nagai; Tomohiro Hasegawa. Vibration isolation analysis and semi-active control of vehicles with connected front and rear suspension dampers, JSAE Review, Vol 18, 1997 , p. 45-50.

5. Lu, Sun. Optimum design of "road-friendly" vehicle suspension systems subjected to rough pavement surfaces. Applied Mathematical Modelling, Vol 26, 2002, p. 635-652.

6. Prentkovskis, $\mathrm{O}$. Interaction between the Vehicle and $\mathrm{Ob}$ stacles: Doctoral Dissertation (Automobilio sq̨veika su kliūtimis: Daktaro disertacija). Vilnius: VGTU, 2000. 117 p. (in Lithuanian).

7. Tamboli, J. A.; Joshi, S. G. Optimum design of a passive suspension system of a vehicle subjected to actual road random excitations. J. Sound Vib., Vol 219, No 2, 1999, p. 193-205.

8. Lukoševičienè, $O$. The accident analysis and simulation. Monogpaph (Autoịvykių analizè ir modeliavimas). Vilnius: Technika, 2001. 244 p. (in Lithuanian).

9. Prentkovskis, O; Bogdevičius, M. Dynamic of a Motor Vehicle Talking into Consideration their Interaction of Wheels and Road Pavement Surface. Transport, Vol XVII, No 6, Vilnius: Technika, , 2002, p. 244-253.

10. Ilarionov, V. A.; Morin, M. M.; Sergeyev N. M. Theory and Design of Automobiles (Теория и конструкция автомобиля). Moscow: Mashinostrojenije, 1985. 368 p. (in Russian).

11. Korn, G.; Korn, T. Reference Book on Mathematics for Scientific Workers and Engineers (Справочник по математике для научных работников и инженеров). Moscow: Nauka, 1968. 720 p. (in Russian).

12. Schick, W; Silverman, G. Fortran 90 and Engineering Computation. John Wiley \& Sons, 1995. 425 p. 